\title{
Neural Maturational Delay as a Link in the Chain of Events Leading to SIDS
}

\author{
Laurence E. Becker
}

\begin{abstract}
Although numerous etiological or triggering factors have been suggested in sudden infant death syndrome (SIDS), the underlying mechanism of death is ultimately cardiac and/or respiratory in nature. As there is no evidence of lung or heart abnormalities, attention has focussed on the neural control of respiration and cardiac function. It is important to appreciate the methodological limitations involved in utilizing autopsy material and the criteria for selection of appropriate controls. This report reviews the pathological evidence for developmental delay in SIDS emphasizing delay of neural maturation of both myelination and synapses. Other abnormalities of the nervous system apparently associated with hypoxia-ischemia such as brainstem astrogliosis are also discussed. The occurrence of SIDS at a precise age together with our preliminary studies indicate that neural developmental delay is an important link in the chain of events leading to SIDS.
\end{abstract}

RÉSUMÉ: Retard de la maturation nerveuse comme lien dans la chaîne d'événements menant au syndrome de la mort soudaine du nourrisson Bien que plusieurs facteurs étiologiques ou déclenchants ont été suggérés comme étant impliqués dans le syndrome de la mort soudaine du nourrisson (SMSN), le mécanisme sous-jacent à la mort est ultimement de nature cardiaque et/ou respiratoire. Comme il n'y a aucune évidence d'anomalie des poumons ou du coeur, l'attention s'est concentrée sur le contrôle nerveux des fonctions respiratoire et cardiaque. II est important d'apprécier les limitations méthodologiques impliquées dans l'utilisation de matérièl d'autopsie et les critères de sélection dans le choix de contrôles appropriés. Ce rapport revoit l'évidence anatomo-pathologique en faveur d'un retard du développement dans le SMSN en soulignant le retard de la maturation nerveuse concernant la myélinisation et les synapses. D'autres anomalies du système nerveux, qui semblent associées à l'hypoxie-ischémie telle que l'astrogliose du tronc cérébral, sont également discutées. L'apparition du SMSN à un âge précis, de même que nos études préliminaires, indiquent que le retard du développement nerveux est un lien important dans la chaîne des événements menant au SMSN.

Can. J. Neurol. Sci 1990: 17:361-37I

Sudden infant death syndrome (SIDS) is the major cause of death in infants between 1 month and 1 year of age. In Canada, the incidence is about 1.2 per 1,000 live births. Since virtually all children with sudden unexpected death in Toronto undergo autopsy at The Hospital for Sick Children (HSC), we have a special opportunity for examining SIDS in the broader context of sudden unexpected death and for standardization of procedure and tissue acquisition. Our findings in Toronto are similar to other centers: peak age 1-4 months, highest incidence in winter, more boys than girls affected. In $10 \%$ of sudden unexpected deaths occurring under 1 year of age, a thorough autopsy rules out SIDS. Diagnoses in these cases include: congenital heart disease, myocarditis, central nervous system trauma (child abuse), cardiomyopathy, encephalitis, meningitis, congenital diaphragmatic hernia and medium chain acyl coenzyme deficiency. Theoretically, there is a subpopulation of SIDS in which the diagnostic criteria are below the threshold of detection: molecular, biochemical or infectious abnormalities may be present, but if there is no apparent impact on morphology, it may be difficult to implicate these abnormalities as the cause of death in SIDS.
Autopsy reveals findings that cause death, contribute to death and/or are incidental to death. If an anatomical cause of death is found, the diagnosis is not SIDS. What remains is the detection of factors contributing to death and incidental to death. In SIDS, with our present inadequate knowledge, it is often difficult to separate these two latter categories.

In SIDS, the mechanism of death must ultimately be related to a respiratory or cardiac phenomenon. If respiratory, the mechanism may be central (either failure of respiratory control or dyskinesia of the respiratory center producing a central type of obstructive apnea) or non-central (respiratory obstruction). However, the cause of any respiratory tract obstruction should be apparent at autopsy. Cardiac dysrhythmias, either centrally or peripherally caused, are also potential mechanisms of death in SIDS.

\section{METhodology}

Neuropathologic observations have focused on evidence to support a "central" mechanism for cardiorespiratory dysfunction in SIDS. As these observations are being made on autopsy

From the Division of Neuropathology (Department of Pathology), The Hospital for Sick Children and the University of Toronto, Toronto

Received March 26, 1990. Accepted May 29, 1990

Reprint requests to: Dr. L.E. Becker, Department of Pathology (Neuropathology), The Hospital for Sick Children, 555 University Avenue, Toronto, Ontario, Canada M5G 1 X8 
material, they must be critically assessed in the light of the considerable limitations imposed by such investigations.

The neural control of cardiorespiratory function is complex and incompletely understood. 1.2 Our knowledge of neuroanatomy is based largely on animal studies, and complexity and change in the structural and chemical bases for cardiorespiratory control of experimental animals during early development is only recently being documented. ${ }^{3}$ In humans, neuroanatomical studies are severely limited by the restricted techniques that can be applied to the nervous system at autopsy.

Within minutes of death, tissue deterioration begins, affecting some parameters more than others. Therefore, the interval between death and autopsy is extremely important. However, as the exact time of death in SIDS victims is often unknown, there is a larger margin of error than in other deaths.

Tissue deterioration brings a hierarchy of decomposition. For example, mitochondrial morphology changes rapidly. However, the identification of astrogliosis does not change significantly for 24 hours or more after death. Autopsy measurements of neurotransmitters, receptors and their messenger RNA will presumably provide rough indications of the status before death. However, there is a danger in pushing highly sophisticated, sensitive techniques beyond the limits imposed by the tissue available for examination. Interpretation of data must take into consideration that the tissue being examined is not ideal.

Further complicating the examination of autopsy tissue in SIDS victims is the vigorous resuscitation now applied to most infants. This has obvious significance in neurotransmitter assays, when ionotopic medication is administered before death.

The methodology and extent of investigation vary from one geographic area to another. Microbiological, biochemical and other types of testing are pursued to different degrees. Isolation of a microbe from the brain without any pathologic reaction would be of dubious significance. How far should investigation be carried out on these cases? Is "routine" bacteriological and virological investigation sufficient? Are viral infections being pursued as vigorously as they should be? Should tissue be frozen for biochemical analysis? Again, an isolated biochemical abnormality may be difficult to interpret. On the other hand, if a specific biochemical abnormality 4 is associated with pathology (e.g., fatty infiltration of the liver, cerebral edema), then SIDS should be excluded as a cause of death.

Pathologists perform autopsies in different ways. How much variability is introduced into the diagnosis of SIDS when several pathologists, forensic pathologists and pediatric pathologists in a variety of institutions pool their cases and submit blocks of lissue to be examined by scientists thousands of miles apart? Traditionally, this is the mechanism investigators use for accumulating sufficient tissue.

Furthermore, to determine whether a subtle observation is normal or abnormal, it must be placed in the context of the variability which exists with normal development. What controls may be used to ascertain the bounds of normal maturation?

The ideal control is a child who dies accidentally (e.g., suffocation), receives no attempted resuscitation and undergoes a complete autopsy within 4 hours of a precisely known time of death. Using only ideal controls, however, would seriously hamper data accumulation not only for SIDS but also for simple developmental parameters such as organ weights. To collect such data, the definition of control has to be compromised. The extent of compromise varies; in fact, the control population is probably the most variable factor among investigators. International standardization of acceptable controls in SIDS research would enormously assist all research scientists. Research in SIDS is complicated by the need for two types of controls: aged-matched controls (conceptional and birth age) for SIDS and controls of different ages for normal development. Effort should be focused on standardization of control populations to be used in SIDS.

\section{Pathology}

Early observations in SIDS victims directed the attention of research investigators to the brain. Steinschneider ${ }^{5}$ noted prolonged periods of apnea in several infants who died of SIDS in a single family, suggesting that SIDS might be caused by abnormal neural control of breathing. Naeye's ${ }^{6}$ report of brain stem astrogliosis in 1976 provided further impetus to study central respiratory control. From our laboratory came the first confirmation of this study, showing significant reactive astrogliosis in areas of the brain stem thought to affect cardiorespiratory control. In addition, numerous respiratory physiology laboratories have reported abnormalities of breathing patterns in groups of infants who are thought to be models of SIDS. 5.8 In most of these cases, central neural mechanisms were postulated. Therefore, neuropathologists became increasingly involved in SIDS research. ${ }^{9}$

Neuropathological studies include observations that may be reactive to hypoxia and/or ischemia, such as astrogliosis of the brainstem, ${ }^{7}$ subcortical leukomalacia and periventricular leukomalacia. ${ }^{10}$ Observations on neurons which obviously produce the essential electrical activity of the neural circuitry are fundamental; however, unlike other cells such as liver cells, the entire extent of most neurons cannot be readily examined. Therefore, visual access to even limited portions of the neuron requires the application of many techniques. A conventional hematoxylin and eosin (HE) stain shows only the perikaryon, which is about $5-10 \%$ of the neuron. To demonstrate dendritic ramifications, Golgi impregnation is necessary. To show axons, Bielschowsky's stain or electron microscopy is required. For the myelin around the axon, Luxol fast blue stains or electron microscopy is utilized.

Neurons communicate with each other at synapses. Although synapses are identified with Golgi's stain and electron microscopy, the species of neurotransmitter/neuromodulator released by the axon terminal is also of anatomical and functional importance: both neurotransmitter and neuropeptide levels in brain and CSF have been reported in isolated cases of SIDS. $11-13$ Functionally related to conventional synapses are the chemoreceptors in carotid body and neuroepithelial bodies. In response to alterations in blood oxygen, carbon dioxide or $\mathrm{pH}$ levels, the chemoreceptor cells release neurotransmitter/neuromodulator to activate the postsynaptic sensory neuron. Alterations in content of neurotransmitter and neuropeptide have been reported in the carotid body 14 and neuroepithelial bodies 15 of infants with SIDS. Other neuropathological observations, such as increased weight of the brain 16.17 and decreased size of the pineal gland 18 are of interest but their cause and possible role in events leading to SIDS are unknown. 
In SIDS, evidence of hypoxic-ischemic insult to the brain includes astrogliosis and subcortical leukomalacia. Evidence of neural maturational delay is indicated primarily under the heading, neuronal assessment. Other observations in carotid body, neuroepithelial bodies, pineal gland and brain growth may be related to developmental delay, but lack of data on normal developmental parameters makes it difficult to be certain.

\section{Astrogliosis}

Scarring in the central nervous system is characterized by astrogliosis, which is a nonspecific reactive process to a wide variety of insults including trauma, infection, tumor, hypoxiaischemia, abnormal metabolites and toxins. In conventional HE preparations, only the nucleus of a normal astrocyte is readily identified. With gliosis, the astrocytic cytoplasm is filled with glial filaments. These are eosinophilic with HE staining but are best stained with the Holzer stain or with Mallory's phosphotungstic acid hematoxylin (PTAH) stain in which the glial fibres stain blue. Although some laboratories have difficulty with these latter two methods, ${ }^{19}$ a good technologist will provide excellent, consistent results. ${ }^{9}$ In fact, preparations using these stains are the basis for many classic neuropathological descriptions. Antibody to glial fibrillary acidic protein (GFAP) using immunoperoxidase methods is the most specific technique and is now the standard astrocytic marker. Ultrastructurally, glial intermediate filaments of reactive astrocytes are indistinguishable from those in normal astrocytes.

When attempting to assess astrogliosis in immature brains, at least two important problems should be recognized: developmental changes occurring in astrocytes and patterns of "normal" background astrogliosis.

During the perinatal period, the number of astrocytes and myelination glia in human brain increases as the glial cells proliferate. Before myelin appears, an increase in glial cells can be detected. In the human internal capsule, for example, the density of glial cells per volume of tissue is six times greater at term than at 24 weeks of gestation. ${ }^{20}$ Similar differences, although to a smaller degree, were found in the hemispheric white matter. ${ }^{21}$ The changing number of GFAP-positive cells (astrocytes and myelination glia) during development is a potential problem in assessing astrogliosis in the brain stem. We observed developmental changes in the GFAP-positive glia of cerebral white matter in the frontal lobes of infants ranging from 28 weeks postconceptional age to 6 years; 21 with age, GFAP-positive processes gradually become larger and the location of the cells shifts from the deep to the superficial white matter. We found similar but more subtle changes in the brain stem occurring before 1 month of age. ${ }^{21}$ In general, the brain stem myelination is more advanced developmentally than the cerebral hemispheres; therefore, shifts in GFAP-positive glia would not bc expected in the brain stem of infants after the neonatal period. Nevertheless, this issue has not been completely resolved.

Astrogliosis in the brain stems of infants with SIDS was first described by Naeye in $1976^{6}$ and confirmed morphometrically by our group. $7,9,22$ Others ${ }^{23-25}$ later made similar observations, although one group ${ }^{19}$ found no astroglial abnormalities. That study, however, is flawed in that post-conceptional ages were not used and only 10 of the 18 SIDS infants were between 2 and 6 months of age.

In our initial studies of brain stem gliosis in $1978,{ }^{7}$ we documented a pattern of background astrogliosis in young infants. Gliosis was present around the circumference of the brain stem in a subpial location, particularly on the lateral surfaces of the brain stem. Subependymal gliosis was also prominent. A small number of astrocytes was present throughout most regions in the brain stem. However, larger numbers of GFAP-positive astrocytes were present in the SIDS infants than in the controls in specific locations (Table 1). Areas in which increased astrogliosis was statistically significant in both term and premature SIDS victims included the reticular nuclei (paramedian, magno-

Table 1: Brainstem Astrogliosis

\begin{tabular}{|c|c|c|c|c|c|c|c|c|c|c|c|c|c|c|c|}
\hline \multirow[b]{2}{*}{ Site } & \multicolumn{5}{|c|}{ Control } & \multicolumn{5}{|c|}{ SIDS (PTAH) } & \multicolumn{5}{|c|}{ SIDS (GFAP) } \\
\hline & - & + & ++ & +++ & $(\mathbf{N})$ & - & + & t+ & +++ & (N) & - & + & ++ & t+t & (N) \\
\hline \multicolumn{16}{|l|}{ Medulla } \\
\hline Midline & 0 & 4 & 5 & 10 & (19) & 0 & 0 & 0 & 21 & (21) & 0 & 3 & 3 & 38 & (44) \\
\hline Magnocellular $r . \mathrm{n}$. & 3 & 15 & 1 & 0 & (19) & 0 & 0 & 14 & 9 & (23) & 2 & 6 & 21 & 17 & (46) \\
\hline Lateral r. n. & 7 & 10 & 2 & 0 & (19) & 0 & 1 & 10 & 12 & (23) & 3 & 5 & 18 & 20 & (46) \\
\hline NTS & 8 & 11 & 0 & 0 & (19) & 0 & 6 & 8 & 9 & (23) & 2 & 10 & 20 & 11 & (43) \\
\hline \multicolumn{16}{|l|}{ Pons } \\
\hline Midline & 1 & 2 & 3 & 13 & (19) & 0 & 0 & 0 & 21 & (21) & 0 & 2 & 3 & 38 & (43) \\
\hline Midline $\mathbf{r}$. . & 6 & 9 & 4 & 0 & (19) & 0 & 7 & 11 & 5 & (23) & 2 & 19 & 15 & 10 & (46) \\
\hline Lateral r. n. & 12 & 7 & 0 & 0 & (19) & 0 & 14 & 7 & 2 & (23) & 2 & 28 & 12 & 4 & (46) \\
\hline \multicolumn{16}{|l|}{ Midbrain } \\
\hline Midline & 0 & 2 & 6 & 11 & (19) & 0 & 0 & 4 & 15 & (19) & 0 & 4 & 7 & 25 & (36) \\
\hline
\end{tabular}

From Takashima et a $1^{7}$ and Becker and Takashima ${ }^{22}$

$$
\begin{aligned}
& r=\text { reticular } \\
& D V N=\text { dorsal vagal nucleus } \\
& +=<5 \text { astrocytes } / 600 \mu \mathrm{m}^{2} \\
& \text { mesen. = mesencephalic }
\end{aligned}
$$

$\mathrm{n}=$ nucleus

ION $=$ inferior olivary nucleus

$++=6-10$ astrocytes $/ 600 \mu \mathrm{m}^{2}$

S.C. $=$ superior colliculus
NTS $=$ nucleus tractus solitarius $-=$ no astrocytes $/ 600{\mu \mathrm{m}^{2}}^{2}$ $+++=>11$ astrocytes $/ 600 \mu \mathrm{m}^{2}$ 
cellular, lateral), the solitary nucleus, the dorsal nucleus of vagus, and the inferior olivary nucleus. Thus, the astrogliosis was most prominent in the tegmentum of the medulla. No statistically significant differences were found in the pons or midbrain of either SIDS group. Because of this apparent geographical distribution of the astrogliosis, we performed postmortem arterio-grams of the medulla to ascertain its degree of vascularity. ${ }^{7}$ The dorsal tegmental region was somewhat hypovascularized compared with other regions of the medulla. Related to this hypovascularity are clinical examples of hypoxia-ischemia occurring in utero or during early infancy, producing gliosis or infarction. ${ }^{26,27}$ These lesions tend to be in the region of the tegmentum in which astrogliosis is most apparent in SIDS victims. This finding could be interpreted to mean that this region of the brain stem is hypoperfused during episodes of bradycardia associated with apnea. ${ }^{28}$

Considering the difficulties in assessing astrogliosis in brains and the probable heterogeneity within SIDS, variability in astroglial counts among different studies is to be expected.6.7.22 More remarkable is the concordance of several reports with respect to the astrogliosis. ${ }^{6.7 .22}$ Although minor differences in astrogliosis may be described in specific nuclei, the astrogliosis of the tegmentum of the medulla has been confirmed by other laboratories.6.17.22-24 Of particular importance in all studies is that the difference between SIDS and controls was apparent only after a significant number of cases was studied. Because astroglial counts overlap with controls, it is not possible to use these counts in an individual case as a diagnostic test of SIDS. It is not clear why some control infants have high astroglial counts in the dorsal tegmentum of the brain stem. ${ }^{22}$

Since gliosis is not specific, it is not the gliosis but what it indicates that is important. Gliosis, like fibrosis, is not a tightly regulated process: if one neuron degenerates, some astrocytes react but the number that become reactive is variable. Counting astrocytes is helpful to provide a rough indication of a scarring process but does not have the same implications as counting neurons. Neuron number is rigidly regulated and specific for precise locations. Neuronal loss suggests decreased function. However, the minimum neuronal loss in specific nuclei sufficient to produce clinically apparent dysfunction has not been determined clinically. In contrast, gliosis per se is indirectly related to dysfunction: when gliosis occurs, neuronal loss is implicated but not always confirmed. Therefore, undue concentration on gliosis may very well be misguided. Who, for example, would count fibroblasts in a renal scar? Counting is only necessary in areas where gliosis is subtle: counting astrocytes around a well-defined cerebral infarction is unnecessary.

The location of the lesion is important to all clinicopathological correlations in neuropathology. However, precise location of astrogliosis is probably less significant than precise identification of neuronal loss. In the functional unit of the nucleus tractus solitarius, scarring in one component implies some dysfunction. Examination of the entire length of the nucleus and identification of an increased number of astrocytes also would indicate dysfunction. This additional information might identify more cases with gliosis but does not provide more insight into pathogenesis. Unlike neuronal counts, total counts of glial cells for entire anatomical regions is uncommon in neuropathology because it is cumbersome, time consuming and often unproductive diagnostically.

\section{Subcortical and Periventricular Leukomalacia}

In the infant brain, the cerebral white matter is vulnerable to ischemic insults. Periventricular leukomalacia occurs in the watershed of the major cerebral arteries. We have also seen a lesion in the subcortical white matter:10,26 it was discrete with no coagulative necrosis and appeared to be a dilution of the white matter with decreased numbers of axons and less myelin than the surrounding normal white matter. Unlike periventricular leukomalacia, there was minimal astrogliosis, rare swollen axons and no infiltration of microglial cells. The pathogenesis of subcortical leukomalacia is unknown. We were able to demonstrate subcortical leukomalacia in $15.5 \%$ of 84 cases of SIDS, 10 $12.7 \%$ of 63 infants with congenital heart disease but only $4.4 \%$ of 45 infants with acute disease. Subcortical leukomalacia did not occur in the neonatal period but had its highest incidence after 1 month of age.

Our hypothesis has been that the subcortical leukomalacia is probably related to a pathogenesis similar to periventricular leukomalacia. With growth, the site of greatest vulnerability may shift and the growing cerebral gyri and the formation of myelin in the subcortical area may create a new area of increased metabolic demand in the slightly older infant. The arterial watersheds at the depth of the sulci in the subcortical U-fibre area are at the end of long cortical branches of the meningeal arteries. ${ }^{10}$ In the immature brain (28 weeks' gestation) with very shallow gyri, the cortico-subcortical medullary branches of the meningeal vessels penetrate the cortex at very regular intervals. In the more mature infant brain (term to 3 months of age) with its deeper sulci, subcortical and medullary arteries from each side of the sulcus bend more acutely at the junction of white matter and extend through the subcortical white matter toward the lateral ventricle. Thus, a triangular area of white matter at the depth of the sulcus occupies a border zone between the subcortical medullary arteries on each side of the sulcus, which may be susceptible to ischemic damage in this area. In older infants, this triangle is better vascularized and less susceptible to ischemia. This developing vasculature and the deepening sulci may be related to the formation of subcortical leukomalacia.

We found that $21.6 \%$ of infants who died of SIDS had either subcortical leukomalacia or periventricular leukomalacia. The incidence was $24.8 \%$ in congenital heart disease and $4.4 \%$ in infants who died of known acute causes. Because subcortical leukomalacia occurs in patients with congenital heart disease, it is tempting to suggest that ischemia produces the subcortical lesion in the infant. This mechanism might also be present in SIDS.

\section{Neuronal Assessment}

The identification of astrogliosis often implies sublethal neuronal damage or neuronal death. Therefore, a neuronal abnormality should occur in the region of astrogliosis, which is the dorsal tegmentum of the medulla. It would be unusual to find neuronal damage without gliosis unless the insult occurred in early gestation. Neurons may be subject to nonfatal or fatal injury. Therefore, a nonfatal injury necessitates assessment of dendritic ramifications, synapses and axons (myelination). Fatal injury requires an assessment of neuron number. Morphometric analysis of neuron numbers in the nuclei of the human brain stem during development is being investigated by Kinney et al. ${ }^{27}$ 
More productive may be the examination of synapses and neurotransmitters despite the biologic limitations imposed by the necessity of using autopsy material. 28

\section{a. Dendrites}

The dendritic branches and their spines are the postsynaptic portion of the neuron. During development, there is excessive production of dendritic ramifications, which are later pruned preserving the dendritic branches that form a functional unit with an ingrowing axon. In the brain stem of cats, Sheibel et al29 reported a changing number of spines with development. That is, many spines were present at birth and the numbers decreased as the cat matured into an adult. Quattrochi et al ${ }^{30,31}$ reported a persistence of spines in SIDS victims and interpreted it as evidence of altered brain stem neural maturation. They examined magnocellular and parvocellular reticular neurons in the paramedial and lateral reticular areas of the medulla and the dorsolateral area of the pons, using rapid Golgi stains. They found the reticular dendritic spines persisted in 17 of 19 SIDS infants while they were lacking on the smooth surface of dendrites in seven of nine control infants. We confirmed these observations in 1985.35 In the medullary reticular formation, a change in dendritic development occurred with increasing gestational age. With normal growth, the dendrites became long, the spines increased in number and their distribution assumed a mature pattern. The number of spines reached a peak at 34-36 weeks' gestation and after birth decreased rapidly (Figure 1).

Term and prematurely born SIDS infants showed persistence of the reticular dendritic spines. This persistence suggested a delay of development to a mature, higher level of neuronal function. Specifically, spine density between 100 and 200 microns from the soma was greater than that of controls in 12 of 14 cases of SIDS. The difference in spine densities was statistically significant in 14 SIDS victims compared with seven controls aged 1-9 months. The spine density in SIDS victims was approximately that of term neonates.

The persistence of dendritic spines in SIDS suggests a delay in maturation of neurons. Because these neurons lie in the region of the brain stem involved in the neural control of cardiorespiratory function, this delay may reflect a functional impairment of the higher levels of respiratory control in SIDS infants. We have found no abnormality of dendritic development in the motor neurons of the inypoglossal nucleus or cervical anterior horn cells in victims of SIDS. ${ }^{33}$

\section{b. Neurotransmitters}

At the synapse, interaction of neurotransmitter with or without a neuromodulator is complex and dynamic. Postmortem studies are static and influenced by premortem status and the interval between death and autopsy. Because of these difficulties,

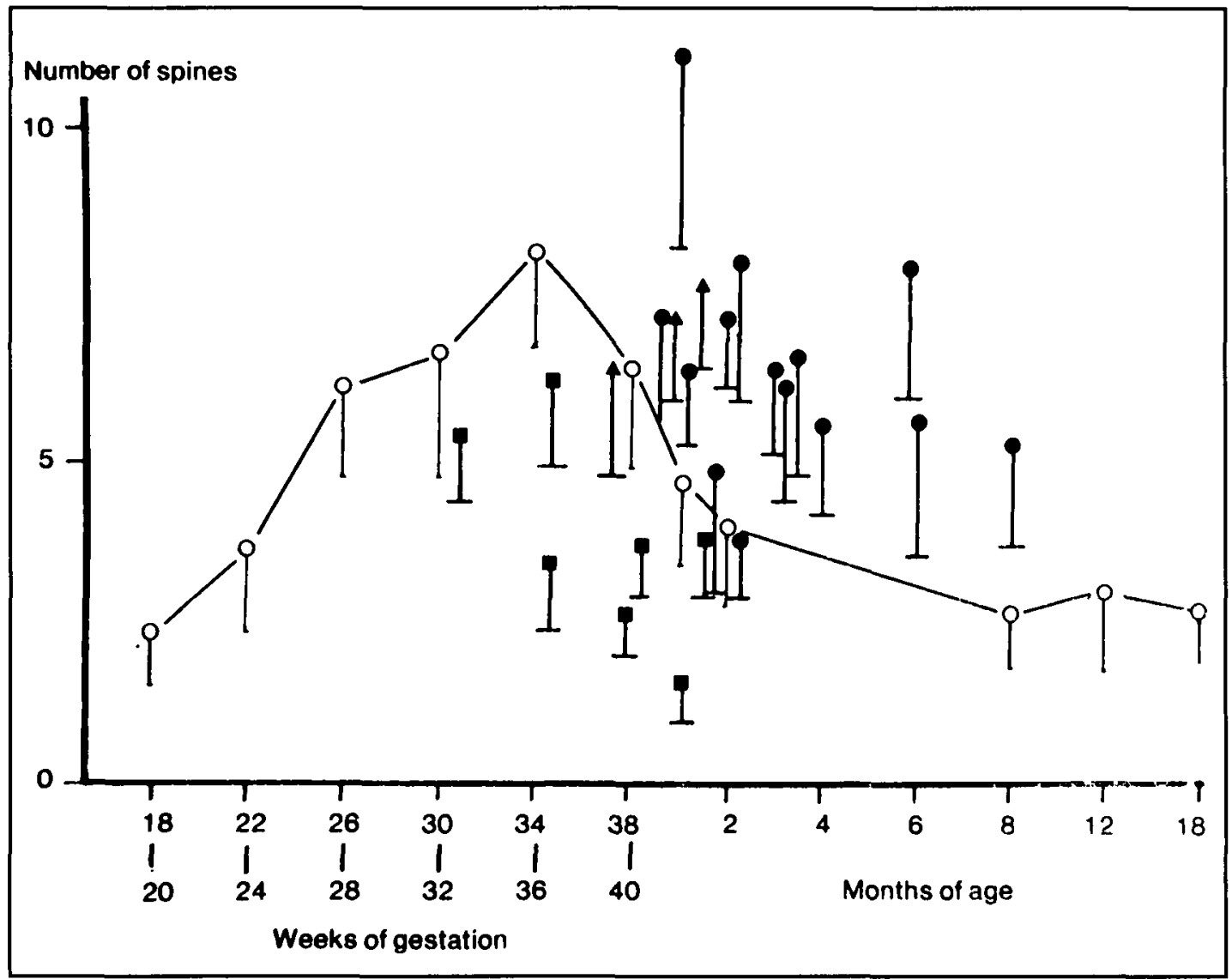

Figure I - Developmental changes of dendritic spine density (number of spines per $25 \mu \mathrm{m}$ ) in the medullary reticular

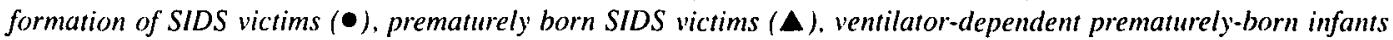
$(\mathbb{B})$ and controls (O). 
studies on the development and maturation of neurotransmitters within the human brain stem have lagged behind experiments in animals. Preliminary animal studies have shown that the interaction of the excitatory and inhibitory neurotransmitters in various brain stem nuclei changes rapidly during late gestation and early infancy. ${ }^{3}$ In animals, it is possible to determine function of the neurotransmitters by administration of agents that excite or inhibit the putative neurotransmitter under investigation. ${ }^{3}$ In human brains, obviously, this kind of manipulation is impossible. However, in infants, levels of neurotransmitters have been determined both in brain and in the cerebrospinal fluid (CSF).

Pearson et al $^{34}$ suggested that, in the human fetus, the catecholaminergic system (tyrosine hydroxylase) was well developed by 10 weeks of age. However, at birth, adult levels of the adrenaline synthesizing enzyme phenylethanolamine- $\mathrm{N}$-methyl transferase (PNMT) were not achieved. ${ }^{35}$ Reflecting developmental changes in neurotransmitter levels in the central nervous system (CNS) are the serotonin decline ${ }^{36}$ and the drop in norepinephrine ${ }^{37}$ during the first few months of life.

A number of reports in the literature suggest the existence of alterations in neurotransmitter levels of catecholamines, beta endorphin, met-enkephalin, and substance P in SIDS.8-12,38-41 The results of many of these studies unfortunately are based on small numbers of SIDS cases and few controls. No developmental studies have been done.

Denoroy et al ${ }^{11}$ reported decreased PNMT in the nucleus retroambiguus, Kolliker fuse nucleus, hypoglossal nucleus and dorsal motor nucleus of the vagus. They also reported decreased dopamine beta hydroxylase in the region of the hypoglossal nucleus and dorsal motor nucleus of the vagus. Ozand and Tildon ${ }^{37}$ found decreased dopamine beta hydroxylase and increased tyrosine hydroxylase in the hypothalamus, putamen and caudate. They found no difference in PNMT levels between these regions and the brain stem.

The endogenous opiate system has received a great deal of attention in SIDS research because of the known effects of opiates on the nervous system. Postmortem analysis showed high concentrations of endorphins in a child with Leigh's encephalopathy 42 who had frequent periods of apnea clinically reversed by naloxone. Pasi et al ${ }^{12}$ showed increased CSF beta endorphin reactivity in infants with apnea and in siblings of victims of SIDS. Subsequently, Kuich and Zimmerman ${ }^{43}$ suggested that SIDS may be caused by an overactivity of the endogenous opiate system. They found that met-enkephalin levels were twice as high in SIDS victims as in controls. However, Lagercrantz et al ${ }^{44}$ could find no difference of met-enkaphalin levels in the medulla between SIDS and controls. In a study by Kozakewich et al ${ }^{45}$ on measuring of CSF met-enkephalin in SIDS, equivocal evidence of met-enkephalin elevation was found, suggesting "hints of but no final proof of excess CSF met-enkephalin in SIDS".

Bergstrom et al 46 reported a significantly higher concentration of substance $P$ in the medulla of SIDS victims but not in other parts of the brain. They suggested that activity in the afferent substance $\mathrm{P}$ containing neurons due to chronic hypoxia was increased.

The difficulty with many of these studies is that the biochemical analysis has not been performed on well-defined neuroanatomical regions. An exception is the microdissection study by Denoroy et al 11,13 who found adrenergic abnormalities in regions of the medulla and pons that are thought to be cardiorespiratory centers. Bradley et al ${ }^{28}$ are embarking on a correlative neurochemical and neuroanatomical approach using receptor autoradiography, which may resolve some of the discrepancies in the literature. This method permits quantitation of neurotransmitter receptor densities in specific areas of the brain.

\section{c. Axons and Myelin}

Myelination is a continuing process starting in the second trimester and continuing well into early childhood. ${ }^{47}$ However, the greatest degree of myelination appears to occur in the first 6 months of life. In the brain, myelin can be assessed by the degree of staining using conventional stains such as Luxol fast blue. In the peripheral nervous system, a more complex system must be established. Morphometric and electron microscopic examinations are essential to determine degree of myelination related to size of axon. 48

In the central nervous system, Kinney et al 49 described regions in the brains of infants with SIDS that appear to be delayed in myelination: temporal lobe at the level of the lateral geniculate body, frontal pole and medial crus pedunculi. They suggest that delayed myelination preferentially affects the late and slowly myelinating tracts but does not correspond to one anatomical region or functional system.

Although a great deal of work has been done on the development of the human sural nerve, there is a paucity of information on development of the phrenic and vagus nerves, which are both directly involved in neural respiratory control.

Phrenic Nerve Although the phrenic nerve of experimental animals has been described in detail, there is no developmental study in infants. We examined the phrenic nerve at postmortem in children aged 3 days to 8 years. ${ }^{50}$ Significant maturation occurs during the first year of life. We found that $43 \%$ of fibres were myelinated at birth and $100 \%$ by the first year. Quantitative developmental studies showed that the myelinated axons doubled in number from birth to the age of 1 year when a plateau was reached. The main period of growth in diameter also corresponded to the first year when the median diameters increased from 1.7 microns at 3 days of age to 3 microns by the eighth month. Unmyelinated axons also grew significantly in the first 11 months when median diameters reached 1.4 microns. There was no significant increase in axonal diameter at later ages. The slope of the regression line for the number of myelin lamellae on axonal diameters increased with age until the sixth month; on the other hand, dispersion around the regression lines decreased in the same period. This suggests a direct relationship between the degree of myelination and the axonal diameter.

In preliminary studies of the phrenic nerve in 15 SIDS cases ranging between 2 and 6 months, the number of myelinated axons and the mean diameter of the axons were measured and compared with five control cases of a similar age.51 There was no significant difference in the number of mean diameter of the myelinated axons. Comparing the number of myelin lamellae to the diameter of the myelinated axons suggested that myelin development was comparable in SIDS infants and controls. Thus, an extensive analysis of phrenic nerve development and a pilot study of the phrenic nerve in SIDS cases have not been able to show any difference between control and SIDS infants. 
Vagus Nerve We studied the development of the vagus nerve in 33 infants and five adults. 52 The age range was from 24 weeks post-conceptional age to 22 years. In infants, the total myelinated vagal fibres increased linearly with the post-conceptional age and, by birth, the total counts were comparable to those of the adults in the 16 term infants but significantly lower in 17 preterm infants. This study indicated that myelination of the vagus nerve was well-developed at birth. Examination of 36 infants who had died of SIDS showed that the mean number of myelinated vagal fibres was not significantly different from that of normal controls. ${ }^{53}$ However, the mean number of small myelinated vagal fibres ( $<2$ microns in diameter) was significantly decreased in the SIDS infants compared with the controls. Also the percentage of small to the total myelinated vagal fibres in the SIDS infants was significantly different from that of controls. These findings suggest that the smaller number of myelinated vagal fibres in SIDS victims may be due to an abnormal or delayed development of vagus. ${ }^{53}$

Of special interest as a potential model of SIDS is our examination of the vagus nerve in persistent infantile sleep apnea (Ondine's curse). ${ }^{54}$ In this infant, who was 2 years old at death, the vagus nerve had fewer small myelinated fibres ( $<2$ microns) than the normal control, as does the vagus nerve in a SIDS victim. However, the total number of all myelinated vagal fibres in the patient with Ondine's curse was significantly less than in the control child, unlike the situation in SIDS where there is no significant difference in the total number of myelinated fibres between SIDS and control cases.

\section{Carotid Body}

The location of the carotid body at the bifurcation of the common carotid artery is ideally suited to determining the composition of arterial blood. Carotid bodies respond reflexly to changes in arterial $\mathrm{pO}_{2}, \mathrm{pCO}_{2}$, and $\mathrm{pH}$ with alterations of respiration. Because of the homeostatic role of carotid bodies and regulation of respiration, the carotid bodies have been of particular interest in determining the pathogenesis of SIDS. Several reports have described the structural abnormalities of the carotid bodies including hyperplasia and hypoplasia. 55 Only one publication has provided ultrastructural evidence for depletion or absence of dense-core neurosecretory granules, ${ }^{56}$ whereas two recent articles 57.58 have not been able to document convincingly a morphometric difference in the number of dense-core neurosecretory granules.

In our study ${ }^{57}$ of the light and electron microscopic findings of carotid bodies from ten victims of SIDS, we assessed the frequency distribution and size of neurosecretory granules. The area of carotid body occupied by the chemoreceptor cells was comparable in the SIDS and control cases. By electron microscopy, carotid body chief cells from both groups contained numerous electron-dense neurosecretory granules. Distribution frequency and size of neurosecretory granules in SIDS cases and controls did not suffer significantly.

Our study showed that the morphology of the carotid bodies from SIDS victims is normal. However, these static morphological studies on postmortem tissue from SIDS victims do not exclude an abnormality of neurotransmitter function on the carotid body. To further pursue the function of the carotid body, we analyzed the catecholamine content of the carotid bodies of SIDS victims and age-matched controls. ${ }^{14}$ In the SIDS infants, the mean values of the three catecholamines, noradrenaline, dopamine and adrenaline were greater than those of the control group except for adrenaline. The most striking difference was an eightfold increase in the concentration of dopamine (Table 2). Dopamine levels in the carotid body showed greater individual variation in SIDS infants than the control infants. Dopamine appeared to be the predominant catecholamine in the SIDS group, but, in the control group, the mean concentrations of dopamine and noradrenaline were approximately equal.

Table 2: Catecholamine Content of Carotid Bodies in SIDS Victims and Control Victims'

\begin{tabular}{lccc}
\hline $\begin{array}{c}\text { Catecholamine } \\
\text { (ng/g tissue) }\end{array}$ & SIDS victims & Controls & $\begin{array}{c}\text { Significance } \\
\mathbf{p}=<\end{array}$ \\
\hline Dopamine & 1622 & 202 & 0.02 \\
Noradrenaline & 502 & 276 & 0.02 \\
Adrenaline & 125 & 47 & 0.10 \\
\hline
\end{tabular}

IModified from Perrin et al. ${ }^{14}$

The finding of elevated dopamine in the carotid bodics of SIDS victims suggests a role in the pathogenesis of the death in SIDS. In both animals and man, dopamine administration inhibits respiration by acting directly on the carotid bodies. 2 Dopamine diminishes the neural discharge from the carotid bodies, which decreases ventilation, particularly the frequency of respiration and tidal volume. Dopamine also appears to inhibit the carotid body response to hypoxia. That is, the finding of high levels of dopamine and raised levels of noradrenaline in the carotid bodies of SIDS victims suggests that these chemosensors may be inhibited in these infants, making them vulnerable to hypoxia.

The carotid body plays an important role in the development of respiratory maturation. In prenatal life, the carotid receptors appear to be of little significance to respiratory activity. At birth, they are not essential for the timing of the first breath. After birth, the chemoreceptors become silent, presumably because of the relative hyperoxia of the milieu. The level of tonic activity of the carotid body appears to reset at a higher level. Therefore, these receptors become responsive to a higher level of arterial $\mathrm{pO}_{2}$.

According to Bureau et al ${ }^{58}$ the postnatal increase in chemosensitivity is responsible for postnatal maturation of breathing. They describe carotid denervation experiments in lambs. 58 One week following the denervation, the lambs are obviously recognizable on the basis of their hypoventilation, hypoxia and hypercapnia. However, of particular interest is the death of three lambs between the fourth and fifth weeks of age. It is suggested that they died during the course of a prolonged apnea during sleep. Also of note was the fact that long-term survivors of the denervation had a reduced growth rate (20\% of that of the lambs with intact carotid bodies at 70 days of age). They concluded that the carotid body receptors play an important role in the postnatal maturation of normal breathing in the lamb and that the lambs became candidates for unexpected death a few weeks after birth because of loss of these receptors. In SIDS, it is still not certain whether the carotid body catecholamine abnormality is the cause or the result of the chronic hypoxia. During chronic hypoxia, the carotid bodies become hyperplastic. Thus, if the increased dopamine levels were secondary to chronic hypoxia, 
the chief cells should be hyperplastic; however, morphologic and morphometric studies of carotid bodies in SIDS show no significant increase in size or volume of chemoreceptor cells. ${ }^{57}$ This suggests that the increased catecholamine content in SIDS may be related to abnormal control of the release of neurotransmitters in the carotid bodies.

It is not clear whether the elevation of dopamine is restricted to the carotid body or represents a more generalized defect in catecholamine metabolism. Haddad et al ${ }^{59}$ suggested an abnormality of autonomic control of respiration affecting heart rate and sleep. In support of this hypothesis are the elevated levels of catecholamine metabolites in the CSF of premature infants with idiopathic apnea. 45,60 In addition, Denoroy et al ${ }^{11,13}$ reported decreased activity of dopamine beta hydroxylase in brain stem nuclei of SIDS infants. The role of the autonomic system in the pathogenesis of SIDS remains uncertain. Pearson et al 25 found no differences between SIDS victims and controls in the numbers of neurons in the sympathetic (superior cervical) and parasympathetic (sphenopalatine) system.

\section{Neuroepithelial Bodies}

Our group has been interested in neuroepithelial bodies as representative of airway chemoreceptors analogous to carotid bodies involved in the autonomic regulation of breathing. Since neuroepithelial bodies are prominent in fetal and neonatal lungs and decline postnatally, they may be particularly important during neonatal respiratory adaptation. Comparison of the immunohistochemical profile, distribution and frequency of neuroepithelial bodies indicates that Bombesin immunoreactive pulmonary neuroendocrine cells and neuroepithelial bodies were higher in the lungs of SIDS victims than in age-matched controls. ${ }^{61}$ This implies activation and hyperplasia of the pulmonary neuroendocrine system in the lungs of SIDS victims. ${ }^{15}$ The factors that contribute to this appearance may be chronic hypoxia or the relative immaturity of the chemoreceptor system in the lungs.

\section{Pineal Gland}

The function of the pineal gland in normal physiology is obscure. Since SIDS occurs during sleep and the pineal gland is thought to influence diurnal rhythm, there may be a relationship. Sparks and Hunsaker ${ }^{18}$ observed that the pineal gland is significantly reduced in weight (morphometrically determined) compared with age-matched controls. The functional significance of this reduction is unknown.

\section{Brain Growth}

In 1976, Naeye et al62 reported delayed development in 28 of 125 children who died of SIDS. They noted a decrease of body weight (40th to 20th percentile) during the first 4 months of life. Jorgensen et al, in $1982,{ }^{63}$ observed a tendency toward slower growth among SIDS victims than living controls. However, the differences were small and individual variations wide, so that growth rate was not considered to be a useful predictor for SIDS.

Our data ${ }^{17}$ show that the brains of SIDS victims born at term were significantly heavier than reference values matched for both age and body length. Male SIDS victims born prematurely had larger brains than controls matched for body length. Similarly, Shaw et al ${ }^{16}$ reported that $81 \%$ of the brains of SIDS victims were above the 95 percentile, including $19 \%$ above the 99.9 percentile. In our series, the children were also tall for their ages. In the report of Shaw's group, ${ }^{16}$ increased brain weight was not obvious before 1 month of age but became evident later. In the subjects that we examined, the raw difference in brain weight seemed to increase with age but this tendency was no longer apparent when values were matched for body length, although SIDS brains remained heavier. It seems, therefore, that the brains did not grow disproportionately after birth but rather that the children were born with larger brains. Data from published tables were used to provide a standard reference population because there are few sources available for organ weights. Even though these tables may include data from SIDS infants, our SIDS population had significantly larger brains and our controls were either similar or smaller.

Children in our series were taller than reference values, in contrast with previous reports suggesting a failure of SIDS victims to thrive: Froggatt et al ${ }^{64}$ and Peterson et al ${ }^{65}$ demonstrated that the weights at death of SIDS infants were between the 10th and 50th percentiles, body growth and length tended to follow the same trend. Jorgensen et al ${ }^{63}$ described a tendency towards slower growth among SIDS victims than living controls. These differences were so small and individual variations so wide that growth rate was not considered to be a useful predictor for SIDS. Although our data suggest that the enlarged brains were present from birth rather than the result of disproportionate postnatal growth, the cause of the increased brain size in SIDS is unknown.

\section{Discussion}

Throughout the world, experience in SIDS consistently emphasizes two observations: SIDS occurs almost always during sleep and most frequently at 1-4 months of age. This suggests a developmentally related pathogenesis involving changes in sleep patterns with respiratory and/or cardiac maturation. Although not well documented, potential causes of delays in maturation include hypoxia, infection, nutrition and perhaps genetics. Assuming that early differentiation and maturation of all neural circuitry depends on precise timing of gene expression, delays in this process could be due to defective genetic control.

Evidence of abnormal maturation is difficult to ascertain because the systemic effects of hypoxia may produce maturational delays and hypoxia may cause insults that mimic maturational delays.

Using morphologic documentation, the changes in the nervous system that suggest retardation of maturation include delayed development of dendritic spines (synapse), 30-32,66 delayed myelination of the vagus nerve 52,53 and delayed central myelination. ${ }^{49}$ Neurotransmitter abnormalities have been reported in SIDS in brain, $11-13$ CSF,45,60 carotid body 14 and neuroepithelial bodies. ${ }^{15}$ Some of the data are reported only in abstracts and some are contradictory. Unfortunately, most studies do not include developmental biochemical investigations. Therefore, it is not possible to place these "abnormalities" in the context of normal maturation in early infancy.

Pathologic observations outside of the nervous system suggesting delayed maturation include the important studies of growth retardation. $65,67,68$ Other specific investigations that may indicate delay in maturation rather than hypoxia are persistence of extramedullary hematopoiesis 6 and persistence of periadrenal brown fat. 6 
Physiological evidence of abnormality in SIDS has been difficult to accumulate. Few experimental models of sudden death in young animals are available. ${ }^{39,58}$ Instead, clinical models have been widely used such as the apnea syndromes (including Ondine's curse), 40,54 siblings of SIDS victims and infants with missed SIDS or ALTE (apparent life-threatening event). 38.41 Although interesting, unfortunately these models are satisfactory only when the infant dies and an anatomical cause of death cannot be found.

Prospective studies should provide the most reliable physiological information on SIDS. Southall et al ${ }^{8,69}$ studied 10,000 infants with 24-hour recordings of electrocardiographs and breathing movements at discharge from hospital and 6 weeks later. In 29 infants a diagnosis of SIDS was subsequently confirmed. Analysis of the recordings showed that SIDS victims did not have prolonged pauses in inspiratory efforts, increased periodic breathing or short apneic pauses. Although identification of infants at risk using measurements of such cardiorespiratory parameters has not been entirely successful, other measurements such as oxygenation must be pursued. 69

Victims of SIDS have also been reported to have higher overall heart rates before death than controls. ${ }^{70.71}$ Heart rates were higher in SIDS victims less than 1 month of age than in controls during all sleep-waking states. SIDS victims more than 1 month old showed higher heart rates during rapid eye movement sleep only. Therefore, as a group, "SIDS victims differ physiologically from control infants and these differences may be especially prominent during particular-sleep-waking states".71

Assuming a developmental delay that includes a delay in the maturation of the cardiorespiratory function, triggering factors sufficient to tip the balance and produce SIDS may be superimposed in a proportion of these infants. The list of factors is potentially long: upper respiratory tract infection, ${ }^{72,73}$ otitis media, sleep irregularities, intestinal Clostridium botulinum, ${ }^{74}$ prolonged production of fetal hemoglobin, ${ }^{75}$ a variation in QT intervals, 31,76 elevated lead burden, ${ }^{77}$ hypothermia ${ }^{78}$ and others.

Complicating the situation is the suggestion that at least some pathologic observations are due to chronic hypoxia (antenatal or postnatal). Naeye made the initial pathologic observations and concluded that tissue changes were due to chronic hypoxia. ${ }^{79}$ Most are non-neuropathologic, such as increased thickening of the smooth muscle in the walls of small pulmonary arteries, increased weight of the free wall of the right ventricle, increased retention of periadrenal brown fat, presence of hepatic erythropoiesis, and increased amount of chromaffin tissue in the adrenal medulla. Markers relevant to neuropathology are increased or decreased glomic tissue in the carotid body, leukomalacia and gliosis in the brain stem. Some changes thought to be produced by hypoxia-ischemia, such as astrogliosis in the brain stem, may be markers of respiratory neuronal injury resulting in a functionally unstable cardiorespiratory system that in some instances leads to SIDS. Of importance are the recent studies showing high postmortem levels of hypoxanthine in the vitreous humor of infants, suggesting antemortem hypoxia. ${ }^{80}$ These studies support a role for hypoxia in the pathogenesis of SIDS. However, the hypoxic hypothesis does not fully explain the timing of the death at 1-4 months of age or the occurrence of death during sleep.

Based on the available data, one may hypothesize a multistep process leading to SIDS. The first step is subtle developmental delay (intra- and/or extrauterine), which must be distinguished from pathologic growth retardation. The second step is an unstable cardiorespiratory-sleep system during 1-4 months of life. Some of these infants will have evidence of chronic hypoxia. This situation must be distinguished from pathologic apneic syndromes. The third step is exposure to triggering factors that contribute to death in SIDS. These must be distinguished from causal factors which are of sufficient degree or extent to cause death on their own. This scenario explains the apparent heterogeneous nature of SIDS, which is due to the variability within each of these "three categories". It also emphasizes the difficulty of investigating SIDS because of the interplay of these factors. Therefore, significant advances in SIDS research will only occur when homogeneous subsets of cases can be selected and analyzed.

\section{Conclusions}

The difficulties associated with using autopsy material for sophisticated neuropathological and non-neuropathologic examination and the problems related to controls (SIDS controls and developmental controls) prevent rapid and convincing progress in understanding SIDS. However, recent evidence suggests that SIDS is the result of a multistage process. Our current approach is highly integrated, incorporating both neuropathologic and systemic observations. Our impression is that sections of the brain or any other system cannot be examined in isolation. Correlations of minor abnormalities in different systems may be more important than irregularities confined to one particular anatomical region. Because of the large number of SIDS cases at our institution, we are using strict criteria to divide cases into term and non-term groups with and without typical features. Histological, histochemical, morphometric, immunohistochemical, electron microscopic and molecular techniques are being applied comprehensively to subsets of SIDS infants using samples of tissue from brain stem, vagus nerve, phrenic nerve, intercostal muscle, diaphragm, carotid body and neuroepithelial bodies. Deviation from control cases and/or developmental curves is considered abnormal. As indicated in this review, published data using similar approaches but applying them to different sets of SIDS suggest that neural developmental delay is an important link in the chain of events that lead to SIDS.

\section{ACKNOWLEDGEMENT}

This study was supported by a grant from National Institute of Child Health and Human Development RO1 HD22713.

The author is grateful for the expert assistance of Ms. Carla Salvador for preparing this manuscript and Ms. Libby Duke for typing it.

\section{REFERENCES}

1. Feldman JL, Smith JC, McCrimmon DR, et al. Generation of respiratory pattern in mammals. In: Cohen A, ed. Neural Control of Rhythmic Movements in Vertebrates. New York: John Wiley \& Sons 1988; 73-100.

2. Bryan AC. Respiratory control. In: Harper RM, Hoffman HJ, eds. Sudden Infant Death Syndrome: Risk Factors and Basic Mechanisms. New York: PMA Publishing 1988; 249-256.

3. Lagercrantz $\mathrm{H}$. Neuromodulators and respiratory control during development. Trends in Neurosci 1987; 10: 368-372.

4. Howat AJ, Bennett MJ, Variend S, et al. Defects of metabolism of fatty acids in the sudden infant death syndrome. $\mathrm{Br}$ Med J 1985; 290 : 177I-1773. 
5. Steinschneider A. Prolonged apnea and the sudden infant death syndrome: clinical and laboratory observations. Pediatrics 1972; 50: 646-654.

6. Naeye RL. Brain-stem and adrenal abnormalities in the suddeninfant-death syndrome. Am J Clin Pathol 1976; 66: 526-530.

7. Takashima S, Armstrong D, Becker L, et al. Cerebral hypoperfusion in the sudden infant death syndrome? Brain gliosis and vasculature. Ann Neurol 1978; 4: 257-262.

8. Southall DP. Role of apnea in the sudden infant death syndrome: a personal view. Pediatrics 1988; 81: 73-84.

9. Becker LE. Neuropathological basis for respiratory dysfunction in sudden infant death syndrome. In: Tildon JT, Roeder LM, Steinschneider A, eds. Sudden Infant Death Syndrome. New York: Academic Press 1983; 99-114.

10. Takashima S, Armstrong D, Becker LE, et al. Cerebral white matter lesions in sudden infant death syndrome. Pediatrics 1978; 62: 155-159.

11. Denoroy L, Kopp N, Gay N, et al. Activités des enzymes de synthèse des catécholamines dans des régions du tronc cérébral au cours de la mort subite du nourrisson. C R Acad Sc Paris t 1980; 291: 217-220.

12. Pasi A, Foletta D, Molz G, et al. Regional levels of $\beta$-lipotropin and $\beta$-endorphin in the brain and hypophysis of victims of sudden infant death syndrome. Arch Pathol Lab Med 1983; 107: 336-337.

13. Denoroy L, Gay N, Gilly R, et al. Catecholamine synthesizing enzyme activity in brainstem areas from victims of sudden infant death syndrome. Neuropediatrics 1987; 18: 187-190.

14. Perrin DG, Cutz E, Becker LE, et al. Sudden infant death syndrome: increased carotid-body dopamine and noradrenaline content. Lancet 1984; 2: 535-537.

15. Cutz E, Chan W, Perrin DG. Hyperplasia of bombesin immunoreactive $(\mathrm{BI})$ cells in lungs of SIDS victims. Lab Invest 1988; 58 (Abstract): $21 \mathrm{~A}$.

16. Shaw CM, Siiebert JR, Heas J, et al. Megalencephaly in sudden infant death syndrome. J Neuropathol Exp Neurol 1987; 46 (Abstract) : 364 .

17. Aranda FJ, Teixeira F, Becker LE. Assessment of growth in cases of sudden infant death syndrome. Neuroepidemiology 1990; 105: 95-105.

18. Sparks, DL, Hunsaker JC III. The pineal gland in sudden infant death syndrome: preliminary observations. J Pineal Res 1988; 5: $111-118$.

19. Pearson J, Brandeis L. Normal aspects of morphometry of brainstem astrocytes, carotid bodies, and ganglia in SIDS. In: Tildon JT, Roeder LM, Steinschneider A, eds. Sudden Infant Death Syndrome. New York: Academic Press 1983; 115-122.

20. Larroche JC, Amakawa $\mathrm{H}$. Glia of myelination and fat deposit during early myelogenesis. Biol Menonate 1973; 22: 421-435.

21. Takashima S, Becker LE. Developmental changes of glial fibrillary acidic protein in cerebral white matter. Arch Neurol 1983; 40: 14-18.

22. Becker LE, Takashima S. Chronic hypoventilation and development of brain stem gliosis. Neuropediatrics 1985; 16: 19-23.

23. Kinney HC, Burger PC, Harrell FE Jr, et al. 'Reactive gliosis' in the medulla oblongata of victims of the sudden infant death syndrome. Pediatrics 1983; 72: 181-187.

24. Ambler MW, Neave C, Sturner WQ. Sudden and unexpected death in infancy and childhood: neuropathologic findings. Am J Forensic Med Pathol 1981; 2: 23-30.

25. Summers CG, Parker VC. The brainstem in sudden infant death syndrome: a postmortem survey. Am J Forensic Med Pathol 1981:2: 121-127.

26. Takashima S, Armstrong DL, Becker LE. Subcortical leukomalacia: relationship to development of the cerebral sulcus and its vascular supply. Arch Neurol 1978; 35: 470-472.

27. Kinney HC, Meagher CC, Simons JE, Matthysse S. Volumetric sampling strategies for heterogeneous brainstem nuclei. $J$ Neuropathol Exp Neurol 1984; 48: 223-244.
28. Bradley B, White WF, Kenney HC. Autoradiographic localization of $[3 \mathrm{H}]$ forskolin binding to adenylate cyclase in the human infant brainstem. J Neuropathol Exp Neurol 1988; 47(Abstract): 317.

29. Scheibel HE, Davies TL, Scheibel AB. Maturation of reticular dendrites: loss of spines and development of bundles. Exp Neurol 1973; 38: 301-310.

30. Quattrochi JJ, Baba N, Liss L, et al. Sudden infant death syndrome (SIDS): a preliminary study of reticular dendritic spines in infants with SIDS. Brain Res 1980; 181: 245-249.

31. Quattrochi JJ, McBride PT, Yates AJ. Brainstem immaturity in sudden infant death syndrome: a quantitative rapid Golgi study of dendritic spines in 95 infants. Brain Res 1985; 325: 39-48.

32. Takashima S, Mito T, Becker LE. Neuronal development in the medullary reticular formation in sudden infant death syndrome and premature infants. Neuropediatrics 1985; 16: 76-79.

33. Takashima S, Mito T, Becker LE. Dendritic development of motor neurons in the cervical anterior horn and hypoglossal nucleus of normal infants and victims of sudden infant death syndrome. Neuropediatrics (in press).

34. Pearson J, Brandeis L, Goldstein M. Appearance of tyrosine hydroxylase immunoreactivity in the human embryo. Dev Neurosci 1980; 3: 140-150.

35. Kopp N, Denoroy L, Renaud B, et al. Distribution of adrenalin synthesizing enzyme activity in the human brain. J Neurol Sci 1979; 41:397-409.

36. Langlais PJ, Walsh FX, Bird ED, et al. CNS monoamine metabolites: correlation with age in normal infants and children. Pediatrics 1985; 75: 580-586.

37. Ozand PT, Tildon JT. Alterations of catecholamine enzymes in several brain regions of victims of sudden infant death syndrome. Life Sci 1983; 321: 1765-1770.

38. Gupta PR, Guilleminault C, Dorfman LJ. Brainstem auditory evoked potentials in near-miss sudden infant death syndrome. J Pediatr 1981; 98: 791-794.

39. Winn K. Sudden infant death syndrome pathology following repeated episodes of anoxia. Lab Invest 1982; 46 (Abstract): 18P-19P.

40. Deykin E, Bauman ML, Kelly DH, et al. Apnea of infancy and subsequent neurologic, cognitive, and behavioral status. Pediatrics 1984; 73: 638-645.

41. Clancy RR, Spitzer AR. Cerebral cortical function in infants at risk for sudden infant death syndrome. Ann Neurol 1985; 18: 41 47

42. Brandt NJ, Terenius L, Jacobsen BB, et al. Hyper-endorphin syndrome in a child with necrotising encephalomyelopathy. New Engl J Med 1980; 303: 914-916.

43. Kuich TE, Zimmerman D. Endorphins, ventilatory control, and sudden infant death syndrome: a review and synthesis. Med Hypothesis 1981; 7: 1231-1240.

44. Lagercrantz H, Bergstrom L, Terenius L. Postmortem analyses of endorphins and substance $P$ in brains from SIDS victims. International Research Conference on the Sudden Infant Death Syndrome, Baltimore 1982.

45. Kozakewich HPW, Vawter GF, Rappaport L, et al. Cerebrospinal fluid met-enkephalin in sudden infant death syndrome. Lab Invest 1984; 50 (Abstract): 6P-7P.

46. Bergstrom L, Lagercrantz H, Terenius L. Post-mortem analysis of neuropeptides in brains from sudden infant death syndrome. Brain Res 1984; 323: 279-285.

47. Brody BA, Kinney HC, Kloman AS, et al. Sequence of central nervous system myelination in human infancy. I. An autopsy study of myelination. J Neuropathol Exp Neurol 1987; 46: 283-301.

48. Schröder JM, Bohl J, von Bardeleben U. Changes of the ratio between myelin thickness and axon diameter in human developing sural, femoral, ulnar, facial and trochlear nerves. Acta Neuropathol 1988; 76: 471-483.

49. Kinney HC, Brody BA, Finkelstein DM, et al. Delayed CNS myelination in victims of the sudden infant death syndrome (SIDS). J Neuropathol Exp Neurol 1988; 47 (Abstract): 370. 
50. Teixeira F, Aranda F, Becker LE. Postnatal development of phrenic nerve: an electron-microscopic morphometric study. J Neuropathol Exp Neurol 1988; 47(Abstract): 313.

51. Becker LE, Teixeira F. Electron microscopic morphometric analysis of the postnatal development of the phrenic nerve. SIDSSymposium, June 9-11, 1988, Graz.

52. Sachis $P$, Becker LE, Armstrong D, et al. Differential delay in myelination of vagal fibres - its relation to apnea of prematurity and the sudden-infant-death syndrome. Arch Dis Child 1979; 54: 152-153.

53. Sachis PN, Armstrong DL, Becker LE, et al. The vagus nerve and sudden infant death syndrome: a morphometric study. J Pediatr 1981; 98: 278-280.

54. Armstrong D, Sachis P, Bryan C, et al. Pathological features of persistent infantile sleep apnea with reference to the pathology of sudden infant death syndrome. Ann Neurol 1982; 12:169-174.

55. Naeye RL, Fisher R, Ryser M, et al. Carotid body in the sudden infant death syndrome. Science 1976; 191: 567-569.

56. Cole S, Lindenberg LB, Galioto FM, et al. Ultrastructural abnormalities of the the carotid body in sudden infant death syndrome. Pediatrics 1979; 63: 13-16.

57. Perrin DG, Cutz E, Becker LE, et al. Ultrastructure of carotid bodies in sudden infant death syndrome. Pediatrics 1984; 73: 646-65I.

58. Bureau MA, Lamarche J, Foulon P, et al. Postnatal maturation of respiration in intact and carotid body-chemodernervated lambs. J Appl Physiol 1985; 59: 869-874.

59. Haddad GG, Epstein MA, Epstein RA, et al. The QT interval in aborted sudden infant death syndrome infants. Pediat Res 1979; 13: 136-138.

60. Myer EC, Morris DL, Adams ML, et al. Increased cerebrospinal fluid $\beta$-endorphin immunoreactivity in infants with apnea and in siblings of victims of sudden infant death syndrome. J Pediatr 1987; 111: 660-666

61. Cutz E, Chan W, Perrin DG. Pulmonary neuroendocrine cells in SIDS: an immunohistochemical and quantitative study. Ann NY Acad Sci 1988; 533: 461-463.

62. Naeye RL, Ladis B, Drage JS. Sudden infant death syndrome: a prospective study. Am J Dis Child 1976; 130: 1207-1210.

63. Jorgensen T, Biering-Sorensens F, Hilden J. Sudden infant death in Copenhagen 1956-1971. IV infant development. Acta Paediatr Scand 1982; 71: 183-189.

64. Froggatt $P$, Lynas MA, Mackenzie G. Epidemiology of sudden unexpected death in infants ("cot death") in Northern Ireland. $\mathrm{Br}$ J Prev Soc Med 1971; 25: 119-134.

65. Peterson DR, Benson EA, Fisher LD, et al. Postnatal growth and the sudden infant death syndrome. Am J Epidemiol 1974; 99: 389-394.
66. Southall DP, Richards JM, Rhoden KJ, et al. Prolonged apnea and cardiac arrhythmias in infants discharged from neonatal intensive care units: failure to predict an increased risk for sudden infant death syndrome. Pediatrics 1982; 70: 844-851.

67. Wilson AJ, Stevens V, Franks CI, et al. Respiratory and heart rate patterns in infants destined to be victims of sudden infant death syndrome: average rates and their variability measured over 24 hours. Br Med J 1985; 290: 497-501.

68. Schechtman VL, Haper RM, Kluge KA, et al. Cardiac and respiratory patterns in normal infants and victims of the sudden infant death syndrome. Sleep 1988; 5: 413-424.

69. Steinschneider A. Nasopharyngitis and the sudden infant death syndrome. Pediatrics 1977; 60: 531-533.

70. Williams AL, Uren, EC, Bretherton L. Respiratory viruses and sudden infant death. Br Med J 1984; 288: 1491-1493.

71. Sonnabend OAR, Sonnabend WFF, Krech U, et al. Continuous microbiological and pathological study of 70 sudden and unexpected infant deaths: toxigenic intestinal clostridium botulinum infection in 9 cases of sudden infant death syndrome. Lancet 1985; 1: 237-241.

72. Giulian GG, Gilbert ES, Moss RL. Elevated fetal hemoglobin levels in sudden infant death syndrome. $N$ Engl J Med 1987; 316: 1122-1126.

73. Weinstein SL, Steinschneider A. QTc and R-R intervals in victims of the sudden infant death syndrome. Am J Dis Child 1985; 139: $987-990$

74. Drasch GA, Kretschmer E, Lochner C. Lead and sudden infant death: investigations on blood samples of SID babies. Eur J Pediatr 1988; 147: 79-84.

75. Dunne KP, Matthews TG. Hypothermia and sudden infant death syndrome. Arch Dis Child 1988; 63: 438-440.

76. Naeye RL. The sudden infant death syndrome: a review of recent advances. Arch Pathol Lab Med 1977; 101: 165-167.

77. Rognum TO, Saugstad OD, Øyaseter S, et al. Elevated levels of hypoxanthine in vitreous humor indicate prolonged cerebral hypoxia in victims of sudden infant death syndrome. Pediatrics 1988; 82: 615-618.

78. Beal MF, Richardson EP, Brandstetter R, et al. Localized brainstem ischemic damage and Ondine's curse after near drowning. Neurology 1983; 33: 717-721.

79. van Belle G, Hoffman HJ, Peterson DR. Intrauterine growth retardation and the sudden infant death syndrome. In: Harper RM, Hoffman HJ, eds. Sudden Infant Death Syndrome: Risk Factors and Basic Mechanisms. New York: PMA Publishing 1988; 203-219.

80. Pereyra PM, Becker LE. Disturbance of growth rate in sudden infant death syndrome. Lab Invest 1989; 60 (Abstract): 6P. 